\title{
Study of Biocompatibility, Mechanical Properties and Microstructural Analysis af Ag-Pd Alloy
}

\author{
Jenifer Vaswani-Reboso, Nestor Florido-Suarez, Pedro Socorro-Perdomo and Julia Mirza-Rosca \\ University of Las Palmas de Gran Canaria, United States \\ Introduction
}

Using biomaterials to reconstruct damaged parts of the human body is a reality, and to do so they must meet a series of conditions and ensure a certain duration. Until relative recently, the biomaterials were basically engineering materials selected on the criteria of their ability to meet specific biological acceptability requirements. Nowadays, many of them are designed, produced, and manufactured with the unique objective of having an applicability in the medical sector [1] [2].

Regarding the alloys for dental use, it was found that, in addition to corrosion resistance, the different alloys with high noble metal content present mechanical and biological properties that are perfectly compatible for oral use. However, due to the economic situation and the consequent increase in the price of gold, the use of these alloys currently has very high costs for a significant fraction of patients. As a solution to this problem, the study of economically alternative alloys is proposed [3].

Due to their attractive properties from a dental point of view and not least due to their reduced price compared to gold, Ag-Pd alloys have been used extensively in prosthetic dentistry in recent years [4]. According to the classification system of the American Dental Association, Ag-Pd alloys are categorized as noble alloys, while Au-based alloys are considered high noble alloys [5].

\section{Experimental}

The study focuses on the alloy Ag-Pd-Cu-Au-Zn-Ir or PALLIAG (commercial name) which, even with a lower content of noble metals, still presents good resistance to corrosion, being suitable for use in the mouth.

The corrosion resistance of metals and their alloys is principally governed by the surface passivation process. In this way, the Palliag alloy should be examined more closely in order to acquire a better understanding of its behavior and to check its viability. Therefore, a study of the physical and mechanical properties will be made by means of metallographic aspects (with Zeiss AxioVert A1 optical microscope) and by means of Vickers hardness (with Buehler microhardness tester). Its electrochemical behavior will also be determined (using a PAR 263A potentiostat-galvanostat connected with a lock-in amplifier PAR 5210), which will allow the comparison of the trends exhibited. It is concluded that the chemical properties are acceptable when compared with reference dental alloys. This locally limits the passivity of the metal, a very small anodic area surrounded by a large cathodic area is generated and as a consequence, local corrosion (pitting) develops rapidly.

Results and discussion

The microstructure of the Palliag alloy after chemically attack is presented in Fig.1. The Vickers hardness was measured at different test loads $(0.001 ; 0.005 ; 0.01,0.025,0.05$ and $0.1 \mathrm{Kg})$ and a Vickers hardness footprint is shown in Fig.2. 
The stabilization potential for the Palliag alloy at $40^{\circ} \mathrm{C}$ is $-0.055 \mathrm{~V}$ and it remains stable for the next 24 hours. These results show that this alloy does not suffer alterations by the electrolyte, this is indicative that they will present low corrosion speeds. This behavior is not indicative that the material is passivated, however, presents a stability that allows use as a dental prosthesis.

It is clear from this that it is a biphasic alloy in which there is an alpha and a beta phase, one soft (approx.110HV) and one hard (approx.180HV). With these obtained results, it is possible confirm that the alloy is homogeneous in all its area and thickness and that there is an absence of different layers superficial, as could be the case of some compact layer in the superficial of thickness considered.

\section{Conclusions}

According to the results obtained during the corrosion potential tests during 30 minutes and 24 hours at room temperature and at $40^{\circ} \mathrm{C}$, it can be concluded the following:

After immersion in Ringer solution, the potential value of the sample studied decreases until it reaches equilibrium, when it stabilizes and remains constant.

The open circuit potential evolution of Ag-Pd is assigned to the passivation of the sample followed by an $\mathrm{Ag}$ superficially enrichment and the potential development of an insoluble $\mathrm{AgCl}$ film on the surface of the alloy.

The corrosion rate of the analyzed sample, calculated by means of Tafel's curves, tells us that its value is 0.012 , which is very good.

It is concluded that the chemical and mechanical properties are acceptable when compared with reference dental alloys.

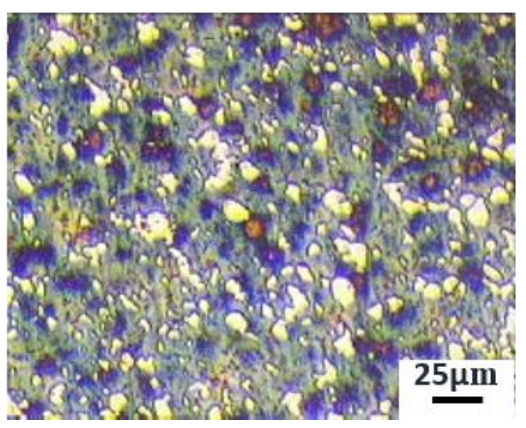

Figure 1. Figure 1. Metallographic analysis polished and chemically attacked surface

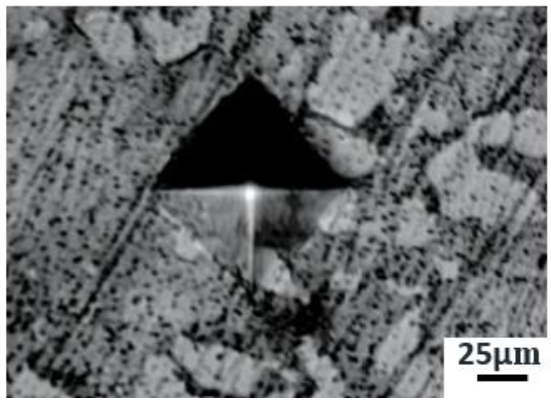

Figure 2. Figure 2. Vickers hardness footprint with $0.025 \mathrm{kgf} \mathrm{load}$ 


\section{References}

[1] M. López Ríos et al., "Effects of nickel content on the microstructure, microhardness and corrosion behavior of high-entropy AlCoCrFeNix alloys," Sci. Rep., 2020, doi: 10.1038/s41598-020-78108-5.

[2] C. M. Garcia-Falcon, T. Gil-Lopez, A. Verdu-Vazquez, and J. C. Mirza-Rosca, "Electrochemical characterization of some cobalt base alloys in Ringer solution," Mater. Chem. Phys., vol. 260, no. September 2020, p. 124164, 2021, doi: 10.1016/j.matchemphys.2020.124164.

[3] G. W. Ali, W. El-Hotaby, B. Hemdan, and W. I. Abdel-Fattah, "Thermosensitive chitosan/phosphate hydrogel-composites fortified with Ag versus Ag@Pd for biomedical applications," Life Sci., 2018, doi: 10.1016/j.lfs.2017.12.021.

[4] A. Lubojanski et al., "Application of selected nanomaterials and ozone in modern clinical dentistry," Nanomaterials. 2021, doi: 10.3390/nano11020259.

[5] A. D. Association, "Glossary of Dental Clinical and Administrative Terms," Gloss. Dent. Clin. Adm. Terms, 2014. 